\title{
Mechanistic Insights into Regulation of JAK2 Tyrosine Kinase
}

\section{Stevan R. Hubbard*}

Department of Biochemistry and Molecular Pharmacology, Skirball Institute of Biomolecular Medicine, New York University School of Medicine, New York, NY, United States

JAK2 is a member of the Janus kinase (JAKs) family of non-receptor protein tyrosine kinases, which includes JAK1-3 and TYK2. JAKs serve as the cytoplasmic signaling components of cytokine receptors and are activated through cytokine-mediated trans-phosphorylation, which leads to receptor phosphorylation and recruitment and phosphorylation of signal transducer and activator of transcription (STAT) proteins. JAKs are unique among tyrosine kinases in that they possess a pseudokinase domain, which is just upstream of the C-terminal tyrosine kinase domain. A wealth of biochemical and clinical data have established that the pseudokinase domain of JAKs is crucial for maintaining a low basal (absence of cytokine) level of tyrosine kinase activity. In particular, gain-offunction mutations in the JAK genes, most frequently, V617F in the pseudokinase domain of JAK2, have been mapped in patients with blood disorders, including myeloproliferative neoplasms and leukemias. Recent structural and biochemical studies have begun to decipher the molecular mechanisms that maintain the basal, low-activity state of JAKs and that, via mutation, lead to constitutive activity and disease. This review will examine these mechanisms and describe how this knowledge could potentially inform drug development efforts aimed at obtaining a mutant (V617F)-selective inhibitor of JAK2.

Keywords: Janus kinases, protein tyrosine kinases, cytokine receptor, autoinhibition, cell signaling and regulation

\section{INTRODUCTION}

Janus kinases (JAKs) are non-receptor protein tyrosine kinases that serve as the catalytic signaling components for a wide range of cytokine receptors, including the receptors for interleukins, interferons, growth hormone, erythropoietin, and leptin (1). There are four mammalian members of the JAK family: JAK1-3 and TYK2 (tyrosine kinase-2), which are constitutively bound to the cytoplasmic region of cytokine receptors. In general, binding of cytokines to the extracellular region of their cognate receptors induces receptor dimerization, facilitating trans-phosphorylation (activation) of the associated JAKs. Typically, the dimerized receptor chains are distinct (e.g., interferon- $\alpha$ receptors 1 and 2) and harbor two different JAK molecules (e.g., JAK1 and TYK2) (2). Cytokine receptors belonging to a subclass that includes erythropoietin receptor (EpoR) and growth hormone receptor (GHR) are homodimeric and bind JAK2 exclusively. For these receptors, it is not entirely clear whether they are dimerized by cytokine or exist as pre-formed, inactive dimers that undergo a cytokine-induced structural rearrangement. Activated JAKs phosphorylate specific tyrosine residues on the cytokine receptors and subsequently on signal transducer and activator of transcription (STAT) proteins (3), which are recruited to the phosphorylated receptors through their SH2 (Src-homology 2) domains. Phosphorylated STATs then translocate to the 
nucleus to initiate specific transcriptional programs. JAK-STAT signaling pathways are critical for organismal development and homeostasis, particularly in immunity $(1,3)$.

JAKs possess four structural domains (Figure 1A): an $\mathrm{N}$-terminal FERM (band 4.1, ezrin, radixin, moesin) domain, an SH2-like (SH2L) domain, a kinase-like or pseudokinase domain [JH2 (Janus homology-2)], and a C-terminal tyrosine kinase domain (JH1). The FERM and SH2L domains form a single structural unit that engages the so-called Box1 and Box2 cytoplasmic regions of cytokine receptors (4) (Figure 1B, left). The SH2L domain of JAKs binds, in its aberrant phosphotyrosine-binding pocket, a glutamate residue in the Box 2 region of the receptor (4-6). As far as is known, receptor binding and specificity are determined solely by the FERM and SH2L domains of JAKs, although the specificity determinants are not fully understood (7).

While homozygous loss-of-function mutations in JAK genes can be lethal (JAK1 and JAK2), or highly debilitating (JAK3, severe combined immunodeficiency), heterozygous gain-offunction mutations in $J A K$ genes can give rise to blood disorders known as myeloproliferative neoplasms (MPNs), which include polycythemia vera, primary myelofibrosis, and essential thrombocythemia, and also to leukemias (9). In a series of papers published in 2005 (10-13), a single point mutation in the pseudokinase domain of JAK2, V617F, was identified in $>90 \%$ of patients with polycythemia vera and in $\sim 50 \%$ of patients with primary myelofibrosis and essential thrombocythemia. Other point mutations, mainly in the pseudokinase domains of JAKs, have also been linked to proliferative blood disorders $(14,15)$.

\section{REGULATION OF JAK2 ACTIVITY}

The tyrosine kinase domain (JH1) of JAKs is responsible for the majority, if not all, of the phosphoryl transfer activity of JAKs (see below regarding JAK2 JH2 activity). In particular, JH1 catalyzes trans-phosphorylation of two tyrosine residues in the kinase activation loop (Tyr1007 and Tyr1008 in JAK2), which stabilizes the active state. Trans-phosphorylation of these two tyrosines is the key step in JAK activation. The activated kinase domain then phosphorylates specific tyrosine residues in the associated cytokine receptor and in the recruited STAT molecules, as well as other tyrosines in the JAK molecule (Figure 1A).

\section{The JAK2 Pseudokinase Domain Negatively Regulates Tyrosine Kinase Activity in the Basal State}

Biochemical studies, in addition to sequencing data from MPN patients mentioned above, have implicated the pseudokinase domain (JH2) of JAKs as a negative regulator of the tyrosine kinase activity of JH1 (16-18) [reviewed in Ref. (19)]. In 2014, the long-sought autoinhibitory interaction between $\mathrm{JH} 2$ and JH1 was elucidated by X-ray crystallography [TYK2 (8)] and, independently, by molecular dynamic simulations [JAK2 (20)]. In this intramolecular (cis) interaction, the $\mathrm{N}$ lobe of $\mathrm{JH} 2$ interacts with the $\mathrm{N}$ lobe and hinge region of JH1 (Figure 1B, right). Importantly, nearly all of the mapped activating mutations in JH2 (e.g., R683G, L611S) and JH1 (e.g., D873N, P933R) lie in this interface, consistent with the hypothesis that destabilization of this interface leads to an increase in JAK2 activation through

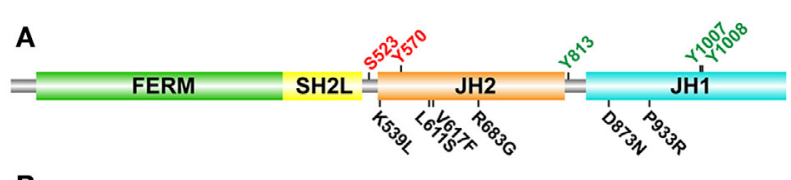

B
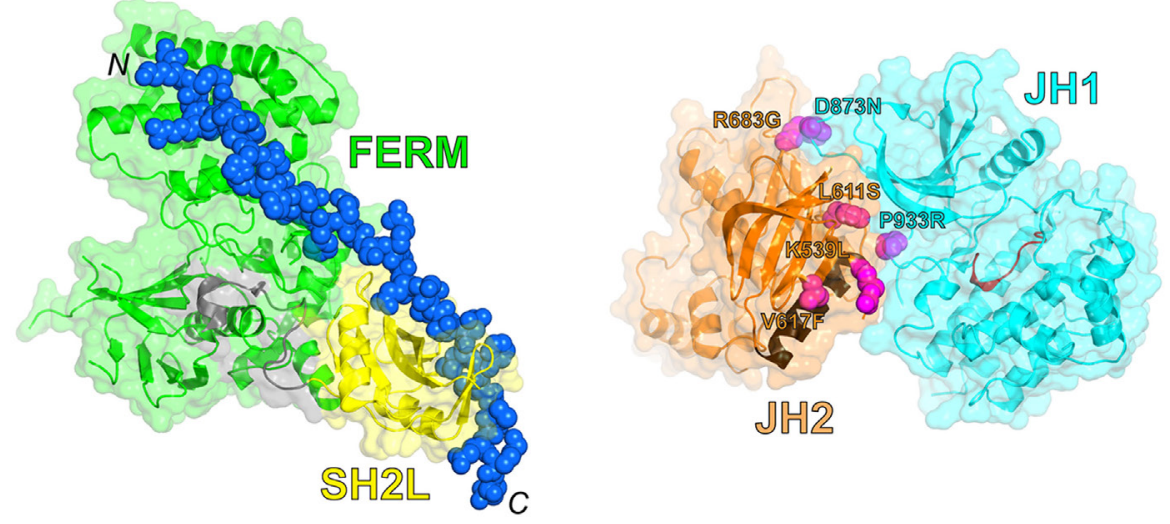

FIGURE 1 | (A) Schematic diagram of the domain organization in JAK2, shown to linear scale (1132 residues in human JAK2). Positive- and negative-regulatory phosphorylation sites (colored green and red, respectively) whose mechanisms are understood are shown above the domains. Select activating mutations in $\mathrm{JH} 2$ and $\mathrm{JH} 1$ are shown below the domains. (B) (Left) Crystal structure of the JAK1 FERM and SH2L domains in complex with a peptide representing the interferon- $\lambda 1$ receptor (6) (PDB code 5L04). The FERM domain is colored green, the SH2L domain is colored yellow, the SH2L-JH2 linker is colored gray, and the receptor peptide (shown in sphere representation; the $\mathrm{N}$ - and C-termini are labeled) is colored blue. (Right) Crystal structure of JH2 and JH1 of TYK2 (8) (PDB code 4OLI). $\mathrm{JH} 2$ is colored orange, with the $\mathrm{C}$ helix colored brown, and $\mathrm{JH} 1$ is colored cyan, with the catalytic loop colored red. The positions of select activating mutations in JAK2, corresponding to those in (A), are shown in sphere representation and colored magenta (TYK2 residues shown, labeled according to the JAK2 mutations). 
increased trans-phosphorylation of JH1. (Conspicuously, V617F is not found in the interface and is discussed below.) How this interaction with $\mathrm{JH} 2$ suppresses the kinase activity of $\mathrm{JH} 1$ is not completely clear, but probably involves physical sequestration of JH1 and/or constraints on JH1 lobe movements that are necessary for phosphoryl transfer activity.

In addition to functioning sterically as a negative regulator of JH1, JAK2 JH2 was shown to possess weak catalytic activity (21), phosphorylating two sites, Ser523 and Tyr570, which had previously been identified as negative-regulatory phosphorylation sites (22-25). It is not well understood how Ser523 and Tyr570 are phosphorylated by JAK2 JH2. According to the crystal structure (26), the end of the activation loop, which forms a short $\alpha$ helix, would seem to impede substrate access to the active site. That is, some rearrangement of the activation loop would be necessary for access. Ser523 was shown to be constitutively phosphorylated in cells (24), whereas Tyr570 has low, but significant, basal phosphorylation, which increases upon cytokine stimulation $(22,23)$, indicating that $\mathrm{JH} 1$ probably also phosphorylates Tyr570 as a negative-feedback mechanism. In vitro studies of JAK2 JH2-JH1 also implicate JH1 as the key kinase domain for phosphorylation of Tyr570 (27). Taken together, these studies suggest that, in vivo, JH2 is responsible for Ser523 phosphorylation and that $\mathrm{JH} 1$ is primarily responsible for Tyr570 phosphorylation.

There are no reports of $\mathrm{JH} 2$ catalytic activity for the other three JAKs, possibly because there are no sites to phosphorylate; Ser523 and Tyr570 are not conserved in JAKs. However, biochemical and structural studies have shown that JAK1, JAK2, and TYK2 are all capable of binding $\operatorname{Mg}$-ATP $(28,29)$. Interestingly, JAK3 contains a lysine (Lys652) in place of the conserved asparagine residue at the end of the catalytic loop. This asparagine in JAK2 and TYK2 JH2, and in bona fide protein kinases, coordinates the ATP-associated $\mathrm{Mg}^{2+}$ ion $(26,29)$, and a lysine at this position might bind to the phosphates of ATP directly, bypassing the requirement for $\mathrm{Mg}^{2+}$. Thus, JAK3 probably binds ATP but without a divalent cation.

Regarding the negative regulatory roles of pSer523 and pTyr570, the model for JAK2 JH2-JH1 derived from molecular dynamics simulations (20) suggests that pSer523 and pTyr570 interact with positively charged residues in JH1 to stabilize the $\mathrm{JH} 2-\mathrm{JH} 1$ autoinhibitory interaction. In general, for JAKs, the $\beta 2-\beta 3$ loop of JH2, where pTyr570 in JAK2 resides, is negatively charged, and the $\mathrm{N}$ lobe of JH1 (interaction site for pTyr570) is positively charged, suggesting that a favorable charge interaction between these two regions stabilizes the $\mathrm{JH} 2-\mathrm{JH} 1$ autoinhibitory interaction for all JAKs.

\section{The JAK2 Pseudokinase Domain Positively Regulates Constitutive Tyrosine Kinase Activity in Pathologic Signaling}

Several biochemical studies have revealed that the role of JAK2 $\mathrm{JH} 2$ in pathologic activation (by point mutation) is complex. That is, loss of the autoinhibitory interaction between $\mathrm{JH} 2$ and JH1 through mutation (e.g., R683G in JH2) is necessary but not sufficient for constitutive activation of JAK2. The first evidence for this came from mutation of Phe595 (to alanine) in the $\mathrm{C}$ helix of $\mathrm{JH} 2$, which suppressed the hyperactivity of V617F (30). Suppression of V617F activity by F595A could be explained by the close spatial relationship ( $\pi$ stacking) between these two phenyalanines (26). Thus, replacement of Phe595 by a less bulky alanine could potentially restore the wild-type local conformation and hence the JH2-JH1 autoinhibitory interaction. However, in the same study (30), F595A also suppressed other activating mutations, for example, R683G, which is $\sim 27 \AA$ from Phe595. This suggests that F595A suppression of R683G (and V617F) hyperactivation is probably due to destabilization of the $\mathrm{C}$ helix. In addition, mutations in the ATP binding pocket of JH2 (which abrogate ATP binding) also suppressed the hyperactivity of various activating mutations (31), presumably because JH2 is less stable without nucleotide bound. Finally, F739R, which is predicted to disrupt the C lobe of JH2 (if not JH2 entirely), also suppressed V617F hyperactivity (26). These mutagenesis data are consistent with a positive role for $\mathrm{JH} 2$ in the pathologic activation of JAK2 by V617F and other activating mutations, and demonstrate that an activating point mutation in $\mathrm{JH} 2$ destabilizes the JH2-JH1 autoinhibitory interaction without compromising the structural integrity of $\mathrm{JH} 2$, particularly in the C-helix region.

A plausible mechanism for the positive role played by $\mathrm{JH} 2$ in pathologic activation is that, when $\mathrm{JH} 2$ autoinhibition of $\mathrm{JH} 1$ (in cis) is weakened by mutation, $\mathrm{JH} 2$ mediates dimerization of JAK2 in the absence of cytokine, which facilitates JH1 transphosphorylation (Figure 2). In essence, pathologic activation of JAK2 might rely on JH2-mediated JAK2 dimerization (cytoplasmic) rather than cytokine-mediated receptor dimerization (extracellular). Although JAK2 $\mathrm{JH} 2$ and $\mathrm{JH} 2-\mathrm{JH} 1$ proteins harboring activating mutations such as V617F are monomeric in solution $(26,27,32)$, full-length JAK2 associated with transmembrane cytokine receptors might behave differently. Receptorassociated JAK2 undergoes quasi-two-dimensional diffusion on the cell surface with some degree of molecular orientation, versus three-dimensional diffusion in the cytoplasm (with random orientation) without receptor. The JAK2 dimerization propensity would be substantially higher in the former case. Indeed, the requirement for cytokine receptor (and for FERM-SH2L, which engages the receptor) for JAK2 V617F hyperactivity (33) can be readily explained by this difference in diffusion properties.

With regard to the nature of the putative JH2-mediated JAK2 dimer, because JH1 of JAK2 (and of other JAKs) has relatively high activity on its own $(8,34,35)$, it is unlikely that $\mathrm{JH} 2$ exerts an allosteric effect on $\mathrm{JH} 1$ in trans (or in cis), although an allosteric activation mechanism in cis between $\mathrm{JH} 2 \mathrm{~V} 617 \mathrm{~F}$ and $\mathrm{JH} 1$ has been proposed (36). Moreover, JH2 would probably not interact with $\mathrm{JH} 1$ in trans as it does in cis (autoinhibitory interaction) due to the presence of the activating mutation (which weakens the JH2-JH1 autoinhibitory interaction) and possibly to steric constraints. Therefore, the positive interaction mediated by JH2 probably involves either JH2 or FERM-SH2L in the other JAK2 molecule (Figure 2). Structural and biochemical evidence point to the $\mathrm{C}$ helix as the likely site of interaction on $\mathrm{JH} 2(26,30,36)$. Although a JH2 dimer was observed in the crystal structure of JAK1 JH2 (32), in which, interestingly, the C helix was in the 


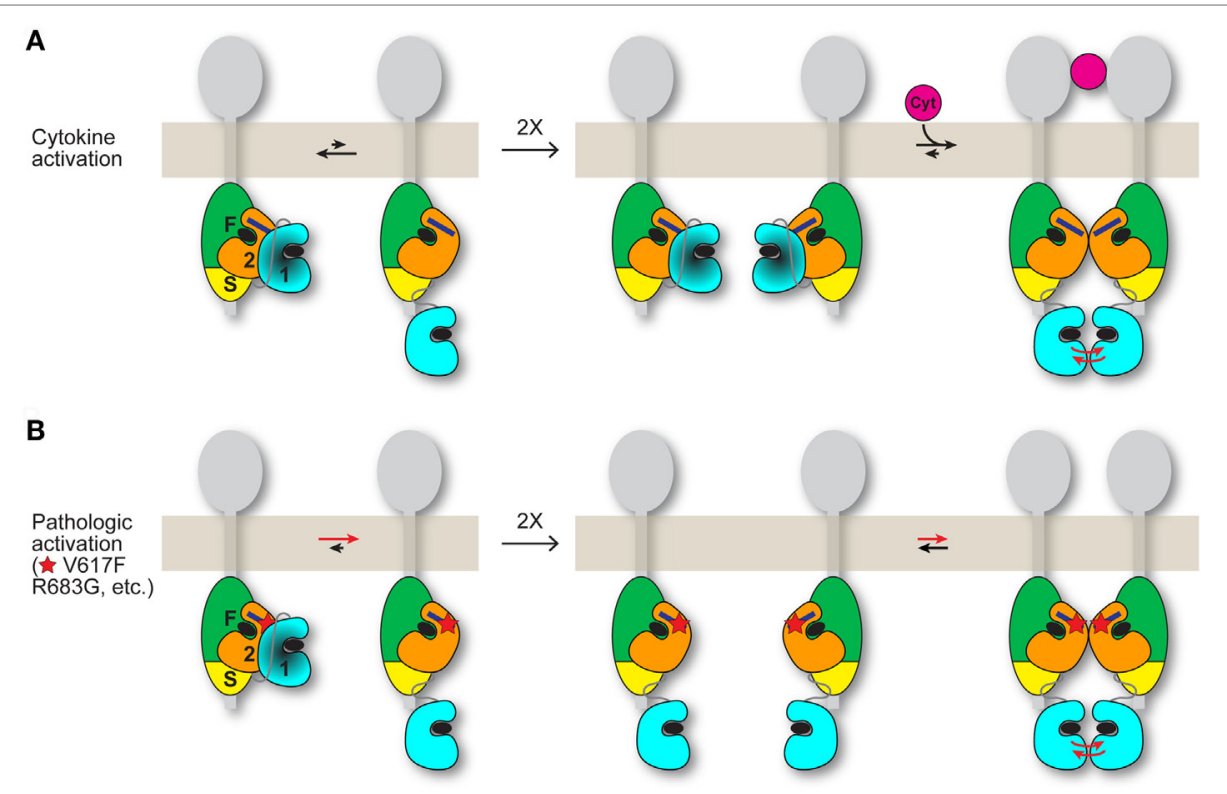

FIGURE 2 | Possible models for JAK2 activation by cytokine and by activating mutation. JAK2 is associated with the cytoplasmic region of a cytokine receptor (gray). The four domains of JAK2 are labeled "F" for FERM, "S" for SH2L, "2" for JH2, and "1" for JH1. The integrated FERM and SH2L domains bind to the cytokine receptor. How JH2-JH1 interacts with FERM-SH2L is not known. The C helix of JH2 is represented by a dark blue rectangle, and ATP is represented by a black ellipse. (A) (Left) An equilibrium (black arrows) exists between the autoinhibited state, in which JH2 exerts an autoinhibitory interaction on JH1 (darkened), and a state in which $\mathrm{JH} 1$ is disengaged from $\mathrm{JH} 2$ and is phosphorylation-competent, with the autoinhibitory state favored for wild-type JAK2. (Middle) In the absence of cytokine, trans-phosphorylation of autoinhibited $\mathrm{JH} 1$ is limited. (Right) Binding of a cytokine (magenta) to the extracellular region of the receptor induces receptor dimerization, which promotes trans-phosphorylation of $\mathrm{JH} 1$ (red arrows). The dimerization process might also include formation of a JAK2 dimer, shown here as a JH2 dimer. (B) (Left) Activating point mutations (shown as a red star) such as V617F and R683G in JH2 shift the equilibrium from the autoinhibitory state to the phosphorylation-competent (and JAK2 dimerization-competent) state. (Middle and right) Mutant JAK2 is capable of dimerizing in the absence of cytokine. Overlap is predicted between the interfaces used by $\mathrm{JH} 2$ for $\mathrm{JH} 1$ autoinhibition and for $\mathrm{JH} 2$-mediated JAK2 dimerization. The activating mutation may directly disrupt the autoinhibitory interaction (e.g., R683G) or hyperstabilize the JAK2 dimer, or both (possibly V617F). Mutations that destabilize the domain structure of JH2, such as those in the ATP binding pocket (31), would suppress activating mutations (V617F, etc.) by destabilizing JAK2 dimer formation, which is necessary for activation in the absence of cytokine. Whether the putative $\mathrm{JH} 2$-mediated JAK2 dimer required for mutational activation in the basal state (B) is the same JAK2 dimer that may form when cytokine dimerizes the receptors (A) is not known. If homodimeric receptors such as EpoR are pre-dimerized in the basal state, then cytokine binding would rearrange the dimer (instead of dimerizing monomeric receptor-JAK2 pairs) to juxtapose the two kinase domains for trans-phosphorylation (A), possibly facilitated by a JH2-mediated interaction. An activating mutation would promote the receptor-JAK2 rearrangement through JH2-mediated JAK2 dimerization in the absence of cytokine binding (B).

center of the dimer interface, mutagenesis studies performed on JAK2 in that study did not support a role for this particular JH2 dimer in activation by V617F.

\section{Possible Mechanisms for Pathologic JAK2 Activation by V617F}

As mentioned above, Val617 in $\mathrm{JH} 2$, the site of the activating mutation (V617F) responsible for the majority of MPNs, is not situated in the JH2-JH1 autoinhibitory interface. The molecular dynamics-based model of the $\mathrm{JH} 2-\mathrm{JH} 1$ autoinhibitory interaction (20) suggests that Val617 is near the SH2L-JH2 linker, which in the model is wedged between JH2 and JH1. The SH2L-JH2 linker was shown to play a role in maintaining JAK2 basal activity (37). Therefore, it is conceivable that substitution of Val617 with bulky residues could displace this linker, decreasing the stability of the JH2-JH1 autoinhibitory interaction. While this may contribute to the constitutive activity of V617F, it is probably not the main activating mechanism. Moreover, in vitro biochemical studies of the tandem kinase domains (JH2-JH1) of JAK2 (27) or TYK2 (8) demonstrated that V617F (V658F in TYK2) does not increase the intrinsic catalytic activity of $\mathrm{JH} 1$.

Based on the crystal structures of wild-type JAK1 JH2 and V658F (V617F-equivalent), Eck and colleagues (32) hypothesized that V617F induces a switch in the conformation of Phe537, which is at the beginning of $\mathrm{JH} 2$, from a tucked position near Phe595 in the C helix to an exposed position. How this conformational switch would lead to activation of JAK2 is not understood, though.

An attractive hypothesis for the mechanism of JAK2 activation via $\mathrm{V} 617 \mathrm{~F}$ is that the mutation hyperstabilizes the putative JH2-mediated JAK2 dimer discussed above (Figure 2), resulting in efficient JH1 trans-phosphorylation. Some support for this mechanism comes from cell-transfection experiments of JAK2 (with two different tags), wild-type or V617F, in which only V617F showed evidence of co-immunoprecipitation (38). Other activating mutations such as R683G, which directly destabilize the $\mathrm{JH} 2-\mathrm{JH} 1$ autoinhibitory interaction, also depend on a structurally sound JH2 (discussed above), but whether V617F and $\mathrm{R} 683 \mathrm{G}$, etc. potentiate formation of the same JAK2 dimer 
or distinct ones is not clear. That E596R in the C helix of JAK2 $\mathrm{JH} 2$ suppresses the hyperactivation of V617F but not of R683G or K539L (36) hints that the dimers might be distinct.

\section{OUTSTANDING MECHANISTIC ISSUES}

Many important mechanistic questions regarding JAK2 activation, both by normal cytokine stimulation and by pathologic mutation, remain unanswered. For example, are class I homodimeric cytokine receptors such as GHR, EpoR, and thrombopoeitin receptor pre-dimerized on the cell surface in the basal state (with non-activated JAK2)? For pre-dimerized receptors, cytokine binding would re-arrange two relatively closely apposed receptor-JAK2 pairs, stimulating JAK2 trans-phosphorylation. Most studies have focused on EpoR, and biochemical evidence for pre-dimerized receptors has been reported $(39,40)$, yet, in the one study that employed in-cell single-molecule fluorescence techniques, EpoR dimers in the basal state were not observed (41). The cell-surface density of expressed receptors is obviously a key parameter in oligomerization studies.

If homodimeric receptors are pre-dimerized in the basal state, an additional question arises whether autoinhibition of JH1 by JH2 occurs in cis (same JAK2 molecule) or in trans (between JAK2 molecules), evidence for the latter (and for pre-dimerized GHR) was reported for JAK2 on GHR (42). Because the JH2-JH1 linker is relatively long ( $\sim 30$ residues in JAK2 and JAK3, $\sim 15$ residues in JAK1 and TYK2), the JH2-JH1 interaction detailed above (Figure 1B, right) could potentially occur in trans rather than in cis. [In the TYK2 JH2-JH1 crystal structure (8), there is a slight cis/trans ambiguity because of the disordered $\mathrm{JH} 2-\mathrm{JH} 1$ linker.] However, the equivalent JAK2 V617F mutations in JAK1 (V658F) and TYK2 (V678F) are also activating, and because JAK1 and TYK2 reside on monomeric receptors in the basal state, the $\mathrm{JH} 2-\mathrm{JH} 1$ interaction would necessarily be in cis for these two JAKs.

Another outstanding question is the nature of the four-domain structure of JAKs in the basal (autoinhibited) state. We know how the FERM and SH2L domains are structurally integrated to bind to cytokine receptors (7) (Figure 1B, left) and how JH2 interacts with JH1 $(8,20)$ (Figure 1B, right), but we do not know the spatial relationship between FERM-SH2L and JH2-JH1. JAK2 $\mathrm{JH} 2-\mathrm{JH} 1$ as expressed in insect cells is active (27), suggesting that autoinhibition requires all four domains. Low-resolution electron micrographs of full-length JAK1 (negative-stained) indicate that the four-domain structure may be loosely assembled (43), which is probably important for the transitioning of JAKs from an autoinhibited state to a phosphorylation-competent state. Finally, the exact mechanisms by which V617F and other

\section{REFERENCES}

1. O'Shea JJ, Holland SM, Staudt LM. JAKs and STATs in immunity, immunodeficiency, and cancer. N Engl J Med (2013) 368:161-70. doi:10.1056/ NEJMra1202117

2. Wang X, Lupardus P, Laporte SL, Garcia KC. Structural biology of shared cytokine receptors. Annu Rev Immunol (2009) 27:29-60. doi:10.1146/annurev. immunol.24.021605.090616 activating mutations lead to constitutive JAK2 activity remain to be determined.

\section{PROSPECTS FOR A MUTANT JAK2 INHIBITOR}

Knowledge of the mechanisms by which JAK2 is activated through normal cytokine binding and by mutation could inform our efforts to design a mutant (V617F)-specific JAK2 inhibitor for the treatment of MPNs. Currently, there is one drug in the clinic, ruxolitinib $\left(\right.$ Jakaf $^{\circledR}$ ), for the treatment of primary myelofibrosis and polycythemia vera. Ruxolitinib is a JAK2 (and JAK1) smallmolecule inhibitor that binds in the ATP binding pocket of JH1. As such, it inhibits the catalytic activity of wild-type JAK2 as well as mutant JAK2, which can lead to side effects such as anemia and thrombocytopenia.

How to target for inhibition JAK2 molecules harboring mutations in JH2 (e.g., V617F) rather than in JH1 poses a difficult conceptual and practical problem (44). If $\mathrm{V} 617 \mathrm{~F}$ and proximal residues in the $\mathrm{C}$ helix of $\mathrm{JH} 2$ are involved in an activating trans interaction (as discussed above), it may be possible to disrupt this interaction with a small molecule that binds to this region, although this region is rather shallow and thus not particularly amenable to targeting by small molecules. Another potential target is the ATP binding pocket of $\mathrm{JH} 2$. It is conceivable that small molecules that bind here could stabilize the JH2-JH1 autoinhibitory interaction, even in a JH2 mutant (V617F), although such molecules could also have the opposite effect of disrupting the $\mathrm{JH} 2-\mathrm{JH} 1$ autoinhibitory interaction and partially activating JAK2. To what extent a small molecule could reshape the ATP binding pocket in $\mathrm{JH} 2$ is also a question, given that the apo and ATP-bound JH2 structures are very similar (26). However, recent studies of compounds that bind in the ATP binding pocket of TYK2 JH2 and inhibit TYK2 signaling provide reason for optimism $(45,46)$. One senses that a wealth of structural data for JAKs will come to light in the next few years, with implications for novel therapeutic modalities.

\section{AUTHOR CONTRIBUTIONS}

The author confirms being the sole contributor of this work and approved it for publication.

\section{FUNDING}

Funding is acknowledged from the National Institutes of Health (R01AI101256, R21AI095808) and from the Myeloproliferative Neoplasm Research Foundation.

3. Levy DE, Darnell JE Jr. Stats: transcriptional control and biological impact. Nat Rev Mol Cell Biol (2002) 3:651-62. doi:10.1038/nrm909

4. Wallweber HJ, Tam C, Franke Y, Starovasnik MA, Lupardus PJ. Structural basis of recognition of interferon-alpha receptor by tyrosine kinase 2. Nat Struct $\mathrm{Mol}$ Biol (2014) 21:443-8. doi:10.1038/nsmb.2807

5. McNally R, Toms AV, Eck MJ. Crystal structure of the FERM-SH2 module of human Jak2. PLoS One (2016) 11:e0156218. doi:10.1371/journal. pone. 0156218 
6. Zhang D, Wlodawer A, Lubkowski J. Crystal structure of a complex of the intracellular domain of interferon lambda receptor 1 (IFNLR1) and the FERM/ SH2 domains of human JAK1. J Mol Biol (2016) 428:4651-68. doi:10.1016/j. jmb.2016.10.005

7. Ferrao R, Lupardus PJ. The Janus kinase (JAK) FERM and SH2 domains: bringing specificity to JAK-receptor interactions. Front Endocrinol (2017) 8:71. doi: $10.3389 /$ fendo.2017.00071

8. Lupardus PJ, Ultsch M, Wallweber H, Bir Kohli P, Johnson AR, Eigenbrot C. Structure of the pseudokinase-kinase domains from protein kinase TYK2 reveals a mechanism for Janus kinase (JAK) autoinhibition. Proc Natl Acad Sci U S A (2014) 111:8025-30. doi:10.1073/pnas.1401180111

9. Vainchenker W, Constantinescu SN. JAK/STAT signaling in hematological malignancies. Oncogene (2013) 32:2601-13. doi:10.1038/onc.2012.347

10. Baxter EJ, Scott LM, Campbell PJ, East C, Fourouclas N, Swanton S, et al. Acquired mutation of the tyrosine kinase JAK2 in human myeloproliferative disorders. Lancet (2005) 365:1054-61. doi:10.1016/S0140-6736(05)71142-9

11. James C, Ugo V, Le Couedic JP, Staerk J, Delhommeau F, Lacout C, et al. A unique clonal JAK2 mutation leading to constitutive signalling causes polycythaemia vera. Nature (2005) 434:1144-8. doi:10.1038/nature03546

12. Kralovics R, Passamonti F, Buser AS, Teo SS, Tiedt R, Passweg JR, et al. A gain-of-function mutation of JAK2 in myeloproliferative disorders. $N$ Engl J Med (2005) 352:1779-90. doi:10.1056/NEJMoa051113

13. Levine RL, Wadleigh M, Cools J, Ebert BL, Wernig G, Huntly BJ, et al. Activating mutation in the tyrosine kinase JAK2 in polycythemia vera, essential thrombocythemia, and myeloid metaplasia with myelofibrosis. Cancer Cell (2005) 7:387-97. doi:10.1016/j.ccr.2005.03.023

14. Haan C, Behrmann I, Haan S. Perspectives for the use of structural information and chemical genetics to develop inhibitors of Janus kinases. J Cell Mol Med (2010) 14:504-27. doi:10.1111/JCMM1018

15. Scott LM. Lymphoid malignancies: another face to the Janus kinases. Blood $\operatorname{Rev}(2013)$ 27:63-70. doi:10.1016/j.blre.2012.12.004

16. Chen M, Cheng A, Candotti F, Zhou YJ, Hymel A, Fasth A, et al. Complex effects of naturally occurring mutations in the JAK3 pseudokinase domain: evidence for interactions between the kinase and pseudokinase domains. Mol Cell Biol (2000) 20:947-56. doi:10.1128/MCB.20.3.947-956.2000

17. Saharinen P, Silvennoinen $O$. The pseudokinase domain is required for suppression of basal activity of Jak2 and Jak3 tyrosine kinases and for cytokineinducible activation of signal transduction. J Biol Chem (2002) 277:47954-63. doi:10.1074/jbc.M205156200

18. Saharinen P, Takaluoma K, Silvennoinen O. Regulation of the Jak2 tyrosine kinase by its pseudokinase domain. Mol Cell Biol (2000) 20:3387-95. doi:10.1128/MCB.20.10.3387-3395.2000

19. Silvennoinen O, Hubbard SR. Molecular insights into regulation of JAK2 in myeloproliferative neoplasms. Blood (2015) 125:3388-92. doi:10.1182/ blood-2015-01-621110

20. Shan Y,Gnanasambandan K, UngureanuD, KimET,Hammaren H, YamashitaK, et al. Molecular basis for pseudokinase-dependent autoinhibition of JAK2 tyrosine kinase. Nat Struct Mol Biol (2014) 21:579-84. doi:10.1038/nsmb.2849

21. Ungureanu D, Wu J, Pekkala T, Niranjan Y, Young C, Jensen ON, et al. The pseudokinase domain of JAK2 is a dual-specificity protein kinase that negatively regulates cytokine signaling. Nat Struct Mol Biol (2011) 18:971-6. doi:10.1038/nsmb.2099

22. Argetsinger LS, Kouadio J-LK, Steen H, Stensballe A, Jensen ON, Carter-Su C. Autophosphorylation of JAK2 on tyrosines 221 and 570 regulates its activity. Mol Cell Biol (2004) 24:4955-67. doi:10.1128/MCB.24.11.4955-4967.2004

23. Feener EP, Rosario F, Dunn SL, Stancheva Z, Myers MG. Tyrosine phosphorylation of Jak2 in the JH2 domain inhibits cytokine signaling. Mol Cell Biol (2004) 24:4968-78. doi:10.1128/MCB.24.11.4968-4978.2004

24. Ishida-Takahashi R, Rosario F, Gong Y, Kopp K, Stancheva Z, Chen X, et al. Phosphorylation of Jak2 on Ser(523) inhibits Jak2-dependent leptin receptor signaling. Mol Cell Biol (2006) 26:4063-73. doi:10.1128/MCB.01589-05

25. Mazurkiewicz-Munoz AM, Argetsinger LS, Kouadio J-LK, Stensballe A, Jensen ON, Cline JM, et al. Phosphorylation of JAK2 at serine 523: a negative regulator of JAK2 that is stimulated by growth hormone and epidermal growth factor. Mol Cell Biol (2006) 26:4052-62. doi:10.1128/MCB.01591-05

26. Bandaranayake RM, Ungureanu D, Shan $Y$, Shaw DE, Silvennoinen $O$, Hubbard SR. Crystal structures of the JAK2 pseudokinase domain and the pathogenic mutant V617F. Nat Struct Mol Biol (2012) 19:754-9. doi:10.1038/ nsmb. 2348
27. Varghese LN, Ungureanu D, Liau NP, Young SN, Laktyushin A, Hammaren H, et al. Mechanistic insights into activation and SOCS3-mediated inhibition of myeloproliferative neoplasm-associated JAK2 mutants from biochemical and structural analyses. Biochem J (2014) 458:395-405. doi:10.1042/BJ20131516

28. Murphy JM, Zhang Q, Young SN, Reese ML, Bailey FP, Eyers PA, et al. A robust methodology to subclassify pseudokinases based on their nucleotide-binding properties. Biochem J (2014) 457:323-34. doi:10.1042/BJ20131174

29. Min X, Ungureanu D, Maxwell S, Hammaren H, Thibault S, Hillert EK, et al. Structural and functional characterization of the $\mathrm{JH} 2$ pseudokinase domain of JAK family tyrosine kinase 2 (TYK2). J Biol Chem (2015) 290:27261-70. doi:10.1074/jbc.M115.672048

30. Dusa A, Mouton C, Pecquet C, Herman M, Constantinescu SN. JAK2 V617F constitutive activation requires JH2 residue F595: a pseudokinase domain target for specific inhibitors. PLoS One (2010) 5:e11157. doi:10.1371/journal. pone. 0011157

31. Hammaren HM, Ungureanu D, Grisouard J, Skoda RC, Hubbard SR, Silvennoinen O. ATP binding to the pseudokinase domain of JAK2 is critical for pathogenic activation. Proc Natl Acad Sci U S A (2015) 112:4642-7. doi:10.1073/pnas.1423201112

32. Toms AV, Deshpande A, McNally R, Jeong Y, Rogers JM, Kim CU, et al. Structure of a pseudokinase-domain switch that controls oncogenic activation of Jak kinases. Nat Struct Mol Biol (2013) 20:1221-3. doi:10.1038/ nsmb. 2673

33. Lu X, Huang LJ-S, Lodish HF. Dimerization by a cytokine receptor is necessary for constitutive activation of JAK2V617F. J Biol Chem (2008) 283(9):5258-66. doi:10.1074/jbc.M707125200

34. Sanz Sanz A, Niranjan Y, Hammaren H, Ungureanu D, Ruijtenbeek R, Touw IP, et al. The JH2 domain and $\mathrm{SH} 2-\mathrm{JH} 2$ linker regulate JAK2 activity: a detailed kinetic analysis of wild type and V617F mutant kinase domains. Biochim Biophys Acta (2014) 1844:1835-41. doi:10.1016/j.bbapap.2014.07.003

35. Zhao L, Ma Y, Seemann J, Huang LJ. A regulating role of the JAK2 FERM domain in hyperactivation of JAK2(V617F). Biochem J (2010) 426:91-8. doi:10.1042/BJ20090615

36. Leroy E, Dusa A, Colau D, Motamedi A, Cahu X, Mouton C, et al. Uncoupling JAK2 V617F activation from cytokine-induced signalling by modulation of JH2 alphaC helix. Biochem J (2016) 473:1579-91. doi:10.1042/BCJ20160085

37. Zhao L, Dong H, Zhang CC, Kinch L, Osawa M, Iacovino M, et al. A JAK2 interdomain linker relays Epo receptor engagement signals to kinase activation. J Biol Chem (2009) 284:26988-98. doi:10.1074/jbc.M109.011387

38. Gorantla SP, Dechow TN, Grundler R, Illert AL, Zum Buschenfelde CM, Kremer M, et al. Oncogenic JAK2V617F requires an intact SH2-like domain for constitutive activation and induction of a myeloproliferative disease in mice. Blood (2010) 116:4600-11. doi:10.1182/blood-2009-07-236133

39. Remy I, Wilson IA, Michnick SW. Erythropoietin receptor activation by a ligand-induced conformation change. Science (1999) 283:990-3. doi:10.1126/ science.283.5404.990

40. Constantinescu SN, Keren T, Socolovsky M, Nam H, Henis YI, Lodish HF. Ligand-independent oligomerization of cell-surface erythropoietin receptor is mediated by the transmembrane domain. Proc Natl Acad Sci U S A (2001) 98:4379-84. doi:10.1073/pnas.081069198

41. Moraga I, Wernig G, Wilmes S, Gryshkova V, Richter CP, Hong WJ, et al. Tuning cytokine receptor signaling by re-orienting dimer geometry with surrogate ligands. Cell (2015) 160:1196-208. doi:10.1016/j.cell.2015.02.011

42. Brooks AJ, Dai W, O'Mara ML, Abankwa D, Chhabra Y, Pelekanos RA, et al. Mechanism of activation of protein kinase JAK2 by the growth hormone receptor. Science (2014) 344:1249783. doi:10.1126/science.1249783

43. Lupardus PJ, Skiniotis G, Rice AJ, Thomas C, Fischer S, Walz T, et al. Structural snapshots of full-length Jak1, a transmembrane gp130/IL-6/IL-6Ralpha cytokine receptor complex, and the receptor-Jak1 holocomplex. Structure (2011) 19:45-55. doi:10.1016/j.str.2010.10.010

44. Leroy E, Constantinescu SN. Rethinking JAK2 inhibition: towards novel strategies of more specific and versatile Janus kinase inhibition. Leukemia (2017) 31:1023-38. doi:10.1038/leu.2017.43

45. Moslin R, Gardner D, Santella J, Zhang Y, Duncia JV, Liu C, et al. Identification of imidazo[1,2-b]pyridazine TYK2 pseudokinase ligands as potent and selective allosteric inhibitors of TYK2 signalling. Med Chem Comm (2017) 8:700-12. doi:10.1039/c6md00560h

46. Tokarski JS, Zupa-Fernandez A, Tredup JA, Pike K, Chang C, Xie D, et al. Tyrosine kinase 2-mediated signal transduction in T lymphocytes is blocked 
by pharmacological stabilization of its pseudokinase domain. J Biol Chem (2015) 290:11061-74. doi:10.1074/jbc.M114.619502

Conflict of Interest Statement: The author declares that the research was conducted in the absence of any commercial or financial relationships that could be construed as a potential conflict of interest.
Copyright $\odot 2018$ Hubbard. This is an open-access article distributed under the terms of the Creative Commons Attribution License (CC BY). The use, distribution or reproduction in other forums is permitted, provided the original author(s) or licensor are credited and that the original publication in this journal is cited, in accordance with accepted academic practice. No use, distribution or reproduction is permitted which does not comply with these terms. 